\title{
Successfully Managed Carotid Endarterectomy with Shunting Under Ultrasound Guided Carotid Sheath Block Combined with Superficial Cervical Plexus Block
}

\author{
๑ Fulya Yılmaz1, ๑ İbrahim Erdinç², ๑ Ahmet Dede1, ๑ Koray Bas³
}

1University of Health Sciences Turkey, İzmir Bozyaka Training and Research Hospital, Clinic of Anaesthesiology and Reanimation, İzmir, Turkey 2University of Health Sciences Turkey, İzmir Bozyaka Training and Research Hospital, Clinic of Cardiovascular Surgery, İzmir, Turkey ${ }^{3}$ University of Health Sciences Turkey, İzmir Bozyaka Training and Research Hospital, Clinic of General Surgery, İzmir, Turkey

\begin{abstract}
Carotid endarterectomy (CEA) is the best treatment option in patients with high grade carotid artery stenosis. The assessment of patient's consciousness in awake patient is still the gold standard for cerebral functions.

Here, we report a case of a 73-year-old man who had left sided weakness 10 days ago. Investigations revealed bilateral carotid stenosis with a $90 \%$ stenosis on the right internal carotid artery and a $60 \%$ stenosis on the left side. He underwent semi-urgent CEA under combined ultrasound guided carotid sheath block (U-CSB) with
\end{abstract}

superficial cervical plexus block (U-SCPB). No additional local anesthetic and/or systemic sedo-analgesic agent(s) were required during surgery.

Application of CSB combined with SCPB, which provided excellent satisfaction for surgeon and patient, can be performed safely and rapidly for CEA under ultrasound guidance. Further studies are needed to demonstrate the reliability and effectiveness of this new technique.

Keywords: Carotid sheath block, superficial cervical plexus block, carotid endarterectomy, shunt

\footnotetext{
Address for Correspondence: Fulya Y1lmaz, University of Health Sciences Turkey, İzmir Bozyaka Training and Research Hospital, Clinic of Anaesthesiology and Reanimation, İzmir, Turkey

e-mail: fulya.dr@gmail.com ORCID: orcid.org/0000-0002-6901-7404

Received: 26.07.2020 Accepted: 26.08.2020
}

Cite this article as: Yılmaz F, Erdinç İ, Dede A, Bas K. Successfully Managed Carotid Endarterectomy with Shunting Under Ultrasound Guided Carotid Sheath Block Combined with Superficial Cervical Plexus Block. EJCM 2020;8(3):152-156.

DOI: 10.32596/ejcm.galenos.2020.07.038

${ }^{\circ}$ Copyright 2020 by Heart and Health Foundation of Turkey (TÜSAV) / E Journal of Cardiovascular Medicine published by Galenos Publishing House. 


\section{Introduction}

Carotid endarterectomy (CEA) is the best treatment option in patients with high grade carotid artery stenosis ${ }^{(1,2)}$, but there is still no consensus on the optimal anesthetic management ${ }^{(1,3)}$. Although so many methods have been used for cerebral function monitoring during general anesthesia, awake patient is still the gold standard ${ }^{(1,2,4)}$. The combination of superficial and deep cervical plexus is a preferred regional anesthesia technique for CEA. Both blocks have their unique complications, but these can be prevented by performing the blocks with ultrasound guidance ${ }^{(3-5)}$.

Both ultrasound guided carotid sheath block (U-CSB) and ultrasound guided superficial cervical plexus block (U-SCPB) are performed rapidly with lower complication rates $^{(1,3,6)}$. Hereby we report a CEA in a patient for whom intraoperative shunt insertion was necessary and it was managed successfully under U-CSB combined with U-SCPB.

\section{Case Report}

A 73-year-old-man $(100 \mathrm{~kg}, 175 \mathrm{~cm})$ with a history of hypertension, ischemic coronary artery disease, benign prostate hypertrophy and smoking was admitted to the emergency department with a left sided weakness 10 days ago. Cranial tomography revealed ischemic stroke and medical therapy was started. Subsequently, he was on clopidogrel therapy. After a week, he was well and physical examination was normal without any sequelae. On further examination, Doppler ultrasound revealed bilateral carotid stenosis with a $90 \%$ stenosis on the right internal carotid artery and a $60 \%$ stenosis on the left side. Semi-urgent CEA under regional anesthesia was planned.

No premedication was administered on the day of the surgery. In the operating room, a peripheral venous line was established and monitoring included peripheral pulseoximetry, 3-lead electrocardiography and invasive blood pressure via contralateral radial artery catheter connected to a monitoring kit. He was resting comfortably with blood pressure of 180/95 mmHg, heart rate of 63 beats/ min, and oxygen saturation at $100 \%$ while breathing $4 \mathrm{~L} /$ min of oxygen by nasal cannula. The patient was placed in supine position with his head turned to the opposite side of the surgical side. Before ultrasound examination, the anatomical landmarks were identified and marked as sternocleidomastoid muscle (SCM), cricoid cartilage, mastoid process. After the skin of the lateral neck was disinfected and sterile covers were applied to the transducer and puncture side, the transducer was positioned to identify the common carotid artery, internal jugular vein and vagus nerve at the level of the $6^{\text {th }}$ cervical vertebra (C6) behind SCM (Figure 1). First U-CSB then U-SCPB application was planned. Under ultrasound visualization, the needle was advanced into the carotid sheath from the posterior border of the SCM transversally. The needle was positioned close to the carotid artery and away from the vagus nerve. $10 \mathrm{~mL}$ local anesthetic (LA) solution (5 $\mathrm{mL} 0.5 \%$ bupivacaine and $5 \mathrm{~mL} 2 \%$ prilocaine) was administered perivascularly and LA spread in a halfmoon figure in the carotid sheath which demonstrated the correct injection (Figure 2). Then, transducer was applied to the anterior border of the SCM at C6 level. 10 mL LA solution (5 $\mathrm{mL} 0.5 \%$ bupivacaine and $5 \mathrm{~mL} 2 \%$ prilocaine) was administered to the posterior border of SCM, superficial to the investing layer of deep cervical fascia under spread of LA was visually assessed on the ultrasound image (Figure 3).

Sensory testing indicated the onset of anesthesia in the appropriate nerve distribution and surgery was started.

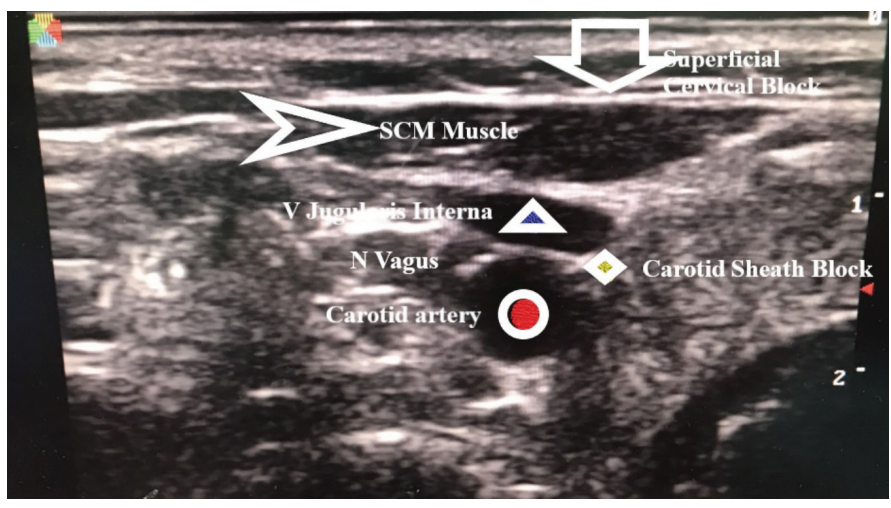

Figure 1. Ultrasonographic anatomic landmarks for superficial cervical plexus block and carotid sheath block SCM: Sternocleidomastoid muscle 
During the dissection of common, external and internal carotid arteries and before clamping of these arteries, heparin (5000 IU) was given intravenously. Clamping was performed three times, but the patient's consciousness deteriorated within 30, 10 and 7 seconds, respectively. So, the surgeon planned intraoperative shunting and carotid shunt was placed (Figure 4). Shunting time, clamping time and overall surgery time were 19, 33 and 55 minutes, respectively. Any additional LA supplementation and systematic sedo-analgesic were not used during the surgery.

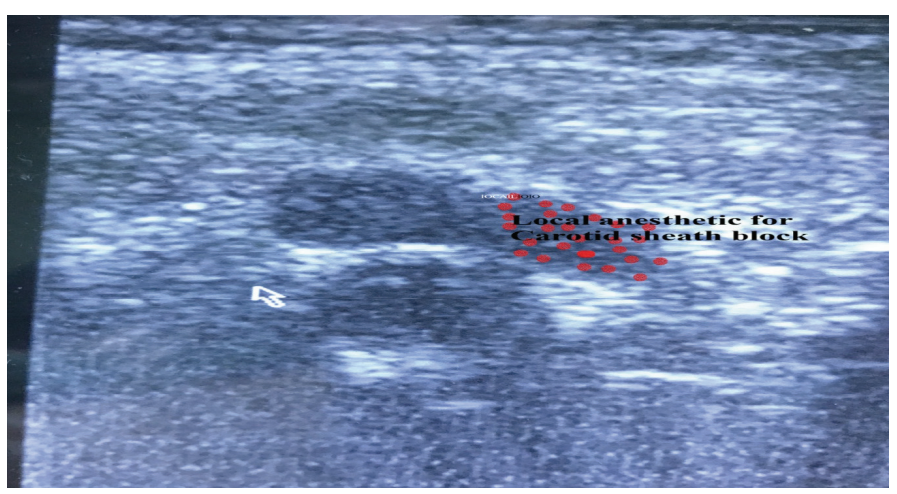

Figure 2. Local anesthetic administered for carotid sheath block

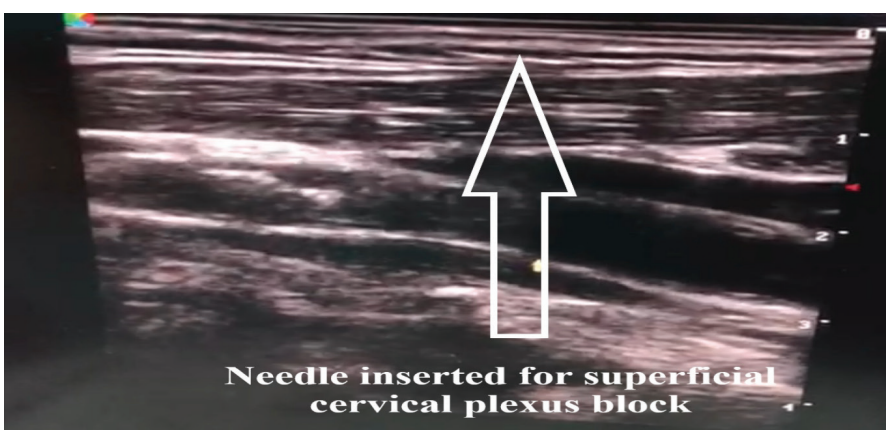

Figure 3. Ultrasound guided cervical plexus block

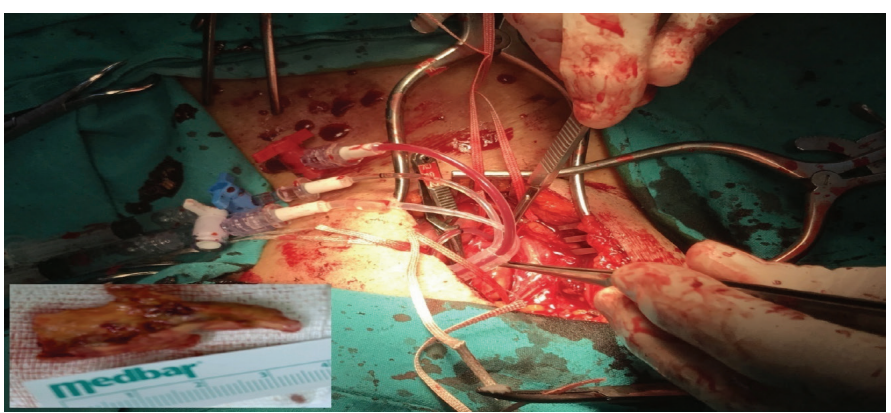

Figure 4. Shunting during CEA and dissected atheroma plaque CEA: Carotid endarterectomy

\section{Discussion}

We perform CEA under combined superficial and deep $\mathrm{CPBs}$ either by conventional technique or under ultrasound guidance in our hospital. Here, we report a CEA in a patient in whom shunt insertion was necessary intraoperatively and it was managed successfully under U-CSB combined with U-SCPB with an excellent satisfaction of anesthesiologist, surgeon and the patient.

In our institution, we prefer to cancel the operation if any vascular injuries occur while performing puncture during blocks. Neither anesthesiologist nor surgeons want to convert to general anesthesia to apply the surgery.

If patient feels pain during the procedure due to the failure of the block, surgeon apply LA to the operation field and/or anesthesiologist prefer to infuse short acting opioid like remifentanil not to affect the evaluation of the consciousness during the clamping.

Superficial CPB is basically a simple subcutaneous injection of LA under the skin, superficial to the investing fascia ${ }^{(1,6)}$, but when it is used alone for CEA, there is a need for supplementation of LA infiltration, especially during the dissection of the distal portion of internal carotid artery ${ }^{(1)}$. So, it must be combined with intermediate, deep or carotid sheath block. Although there are so many studies for CEA under the combination of SCPB, Intermediate Cervical Plexus Block (I-CPB) and/or Deep Cervical Plexus Block (D-CPB), there are only a few studies with combination with CSB. Carotid sheath surrounds the internal jugular vein, carotid artery and vagus nerve. Injection of LA near to the carotid artery by US-guidance in carotid sheath was named as "perivascular regional anesthesia" by Rössel et al. (3), "carotid sheath block" by Casutt et al. ${ }^{(4)}$ and "locoregional anaesthesia" by Martusevicius et al. ${ }^{(5)}$. These studies have reported that LA spread in a half-moon figure in the carotid sheath demonstrates the correct injection and supply sufficient anesthesia. It is not necessary that LA must surround the carotid artery for U-CSB. 
Application of CPBs under ultrasound guidance can reduce the LA dose administered for blocks and prevent block associated complications ${ }^{(3,6)}$. Also, according to the guidelines, it is safer to perform cervical blocks under ultrasound guidance for CEA in patients who are under anticoagulants or antiaggregant therapy. However, neither conventional nor ultrasound guided CPB prevents inadequate anesthesia especially in neurovascular sheath region. It can be explained by the tissues around the carotid artery not only innervated by cervical plexus but also by branches of vagus and glossopharyngeal nerves ${ }^{(3)}$.

Rössel et al. ${ }^{(3)}$ performed ultrasound guided perivascular regional anesthesia combined with I-CPB for 34 patients undergoing CEA. They reported that this combined technique was effective for CEA. Mądro et al. ${ }^{(2)}$ compared infiltration anesthesia with ultrasound guided ICPB combined with carotid sheath infiltration for CEA. They reported that combined block improved patient's and surgeon's comfort, wassafer, relatively simple, and easy to master, required little time to perform. Martusevicius et al. ${ }^{(5)}$ reported that ultrasound guided locoregional anesthesia for CEA provided good quality analgesia with a limited need for intraoperative LA supplementation. Casutt et al. ${ }^{(4)}$ showed the spread of LA following carotid sheath block by computed tomography scan of the head, neck region and upper thorax. They reported that LA spread extensively in carotid sheath and ring formation of LA around the artery did not seem necessary for successful anesthesia for CEA.

Although authors name the same block differently, all have reported that carotid sheath block is safe, simple, can be performed rapidly, sufficient for surgery, requires lower supplemental LA during surgery and is an alternative approach with lower complication rate ${ }^{(1-5)}$.

In the studies, it was combined with either superficial or intermediate $\mathrm{CPB}^{(2,3)}$. If it is applied alone, intraoperative supplementation of LA increases because carotid sheath is like an envelope and LA applied for CPB may not block the branches of vagus nerve which requires LA supplementation during the dissection of the carotis.
Due to the limited number of publications in the literature, there is no clear information about which local anesthesia and at what doses should be used. We preferred the combination of bupivacaine with prilocaine for rapid action and long duration.

In conclusion, in our case, the application of U-CSB combined with U-SCPB was performed rapidly, and supplied sufficient anesthesia for CEA that necessitated shunting intraoperatively. We thought that this combination for CEA might reduce supplemental LA, complications associated with additional LA and helped to avoid D-CPB specific complications. Further studies are needed to demonstrate the reliability and effectiveness of this new technique.

\section{Ethics}

Informed Consent: Written informed consent of the patient was taken.

Peer-review: Externally peer-reviewed.

\section{Authorship Contributions}

Surgical and Medical Practices: F.Y., İ.E., K.B., Concept: F.Y., Design: F.Y., İ.E., Data Collection or Processing: F.Y., İ.E., A.D., Analysis or Interpretation: F.Y., A.D., K.B., Literature Search: F.Y., K.B., Writing: F.Y., K.B.

Conflict of Interest: There is no conflict of interest between the authors.

Financial Disclosure: There is no financial support for this case report.

\section{References}

1. Yilmaz F. Anesthesia Management for Carotid Endarterectomy: Review article. EJCM 2019;7:50-9.

2. Mądro P, Dąbrowska A, Jarecki J, Garba P. Anaesthesia for carotid endarterectomy. Ultrasound-guided superficial/intermediate cervical plexus block combined with carotid sheath infiltration. Anaesthesiol Intensive Ther 2016;48:234-8.

3. Rössel T, Kerstring S, Heller AR, Koch T. Combination of high-resolution ultrasound-guided perivascular regional anesthesia of the internal carotid artery and intermediate cervical plexus block for carotid surgery. Ultrasound in Med Biol 2013;39:981-6. 
4. Casutt M, Breitenmoser I, Werner L, Seelos R, Konrad C. Ultrasoundguided carotid sheath block for carotid endarterectomy: a case series of the spread of injectate. Heart Lung and Vessel 2015;7:168-76.

5. Martusevicius R, Swiatek F, Joergensen LG, Nielsen HB. Ultrasoundguided Locoregional Anaesthesia for Carotid Endarterectomy: A Prospective Observational Study. Eur J Vasc Endovasc Surg 2012;44:27-30.
6. Yilmaz F, Bas K, Ulugolge B. A request for a clarification about classification and nomenclature of cervical plexus blocks. Ann Med Res 2019;26:966-7. 